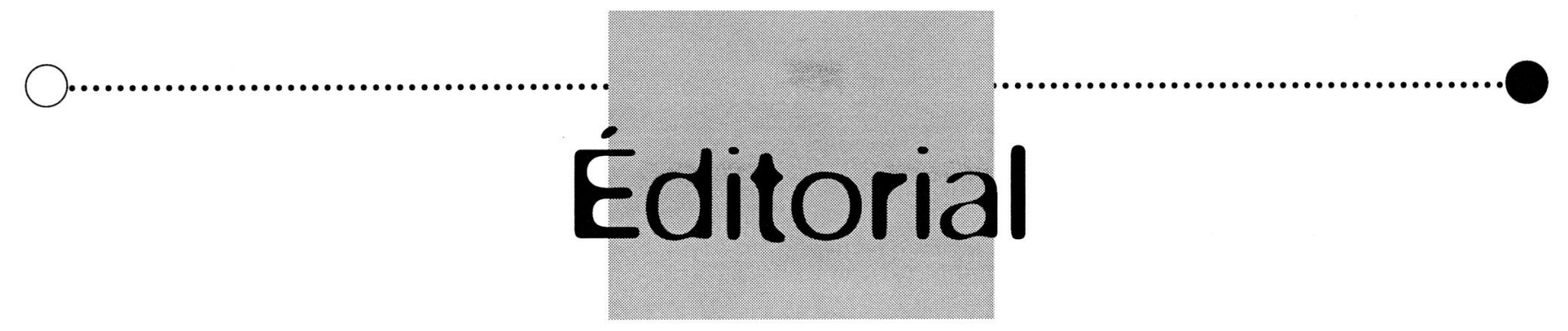

\title{
Psychiatrie et société : nouveaux contrats, nouvelles frontières
}

\section{Psychiatry and society : new deal, new frontier}

\author{
Marc GROHENS*
}

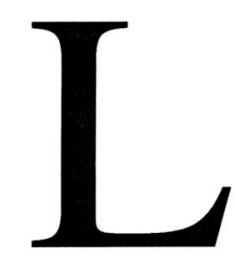

a place de la psychiatrie au sein de la société est en pleine mouvance. Pourquoi ce titre? L'instabilité actuelle d'une société qui voit ses valeurs remises en cause oblige à une recomposition de la place de la psychiatrie et des psychiatres au cœur de notre espace contemporain. Cela n'a peut-être pas été notre choix mais, que nous le voulions ou non, c'est une contrainte sur le questionnement de l'ordre post-moderne, celui qui a succédé après la grande déstabilisation des trente glorieuses, et c'est devenu une préoccupation actuelle.

En effet, événement marquant, la santé mentale a occupé le devant de la scène politique française aux États Généraux de Montpellier et le débat passionné sur l'amendement Accoyer a largement mobilisé les professionnels et suscité l'intérêt des médias assurant une large diffusion auprès de tous les publics.

De nombreux soubresauts ont ainsi émaillé le champ de la psychiatrie, élargissant ce qui était sa mission de lutter contre les maladies mentales pour s'occuper de prévenir et traiter de tout ce qui se rapporte à la souffrance psychologique dans tous les domaines de la société. Les questions, qui touchent autant à l'orientation de la politique de santé qu'aux moyens d'apporter des réponses, débordent donc largement le simple champ des spécialis- tes ou des spécialités. Tous les secteurs de la vie sociale et de la société sont concernés ainsi que leurs différents acteurs, rassemblant dans une dimension qui pourrait parfois paraître hétéroclite, les travailleurs sociaux, les politiques, les associations, les syndicats, les médecins et psychologues du travail, les usagers du système de santé, les entreprises...

La période contemporaine est en effet marquée par l'événementiel, l'instantané, le sécuritaire et la consommation. Ainsi, nous sommes régulièrement informés de l'augmentation de la consommation de soins et, corollaire, du «trou » du système d'assurance sociale, qu'il convient d'ailleurs dans l'ordre de réformer et de combler, sans pour autant que la question soit posée directement à nous tous concernés. Il n'est donc pas vain de constater que la question de la santé, et pour ce qui nous occupe de la santé mentale, a débordé le cadre du discours et du savoir psychiatrique pour poser les jalons de nouveaux contrats échappant aux limites déjà exposées du cadre psychiatrique et du simple processus et raisonnement médical.

Regardons ensemble ce qui nous est demandé, proposé ou imposé.

Les usagers : la loi de mars 2002, qui a bien sûr englobé la psychiatrie, a refondé la relation médecin-malade. La psychiatrie y est d'emblée incluse et n'est pas devenue une exception mais 
a au contraire connu une dialectique nouvelle autour de la relation avec les familles, la personne de confiance et les équipes. Ce renouvellement du discours et du travail autour du dossier médical, de la prescription, du contrat de soins participe à notre sens à la déstigmatisation de la maladie mentale et à l'extension de l'appropriation d'une relation posée et plus paisible entre les différents partenaires impliqués.

Le partenariat : cette dimension s'est largement étoffée malgré le recours trop fréquent, dans le domaine de l'expression publique, à des mots-clés (comme « réseau ») pas toujours suivis d'effets sur le terrain, faute de moyens financiers et humains.

Les faits démontrent cependant une réelle inscription de ces pratiques transversales qui poursuivent le travail de décloisonnement initié par la création du secteur il y a déjà quarante ans. Le risque serait plutôt que les « pratiques de réseau » ne deviennent le cache-misère du manque de psychiatres en dehors des métropoles françaises et du réseau TGV, nous renvoyant à une psychiatrie des champs et des villes nourrissant une succession de dichotomies manichéennes.

La technicité et l'expertal : ces aspects reviennent au détour de questions de société ramenées sans cesse au-devant de l'actualité. Le traitement des agresseurs sexuels oblige la profession à un positionnement qui doit être clair : c'est bel et bien à la société qu'il appartient de définir ce qu'est l'ordre moral et les valeurs qui soutiennent les règles de vie dans cette dernière. Un saut de registre ne saurait que nuire à la transparence et à la définition de notre métier qui relève tant de l'art que de la science au service du malade. Cette recomposition confondant le registre du pervers, du « fou » et du normal au nom de la médicalisation d'un tel raccourci ne peut que creuser le fossé entre les différents acteurs concernés, les mettant au défi de remèdes impossibles et d'attentes toujours décevantes. Il nous appartient là encore en tant que professionnels d'accompagner notre discours pédagogique d'une attitude ferme et consensuelle pour ce qui est de nos possibilités de soins, et plus largement d'intervention, au regard d'une nouvelle tentation de contrôle social.

Le remboursement et la remise en question d'une médecine accessible à tous deviennent un réel enjeu qui nécessite le rappel des avancées depuis 1'après-guerre. La lente agonie d'un système basé sur la proximité, la qualité constante, au nom d'une efficience basée sur une critériologie importée d'outre-Atlantique, doit alerter et soulever une conscience citoyenne pas toujours en éveil.
La question de la surconsommation de psychotropes dans la population française ne cesse d'interpeller comme le nouvel impact des multiples conduites addictives, le dernier avatar étant peut-être une addiction « au bonheur » se contre-balançant au nouveau paradigme de la dépression, qu'A. Ehrenberg décrit comme la pathologie d'une société individualiste et démocratique, où l'individu est écrasé par 1'impératif contemporain d'affirmation individuelle. Corollaire à ces nombreuses questions et difficultés, la demande de soins psychologiques et psychiatriques explose à tel point que leur « régulation » interpelle. Quel domaine ou secteur de la société échappe à ces nouvelles aspirations au bien être et donc au traitement et à la prévention de son opposé le mal être ?

De nouvelles perspectives se sont ainsi ouvertes, courant de la victimologie au harcèlement moral et à l'exclusion. Elles se sont clairement installées, obligeant à une recomposition et un repositionnement des intervenants psychiatriques au minimum.

Sans pour autant procéder à un inventaire à la Prévert, il nous parait ici primordial de rappeler l'irruption des violences dans le cadre de nos institutions et, plus largement, dans ce qui touche aux fondements de notre relation soignante. Les événements de Pau, après les discours se voulant rassurants des autorités, nous obligent à une réflexion sur la lente et inexorable montée des violences dans notre quotidien. Aussi sommes-nous directement interpellés à plusieurs titres sur le rôle supposé qui nous incombe et sur les propositions de solutions qu'il nous faut fournir dans les références qui sont nôtres et qui renvoient à une vision plus globale et multidimensionnelle du sujet malade et de l'individu.

Notre propos ne vise pas à l'exhaustivité mais à un positionnement réflexif.

Crise des valeurs qui se condense sur des aspirations revendiquant le droit au bonheur individuel au détriment du partage et de la solidarité communautaire et républicaine, il règne sur la société actuelle de nouvelles exigences réglementaires qui, au regard de l'histoire, pourraient paraître liberticides au nom d'une sorte de "pax psychiatrica » extension de l'apaisement social. Il nous appartient, et c'est à la fois notre chance et devoir, de redéfinir les fondements de l'alliance thérapeutique qui nous unit à celui et ceux qui souffrent, en gardant à l'esprit cette modestie de notre regard et pratique qu'est « primum non nocere ». New deal, new frontier donc ! 